\title{
Computational Design and Analysis of a Multi-epitope Against Influenza A virus
}

\author{
Samaneh Rostaminia ${ }^{1} \cdot$ Seyed Soheil Aghaei ${ }^{1} \cdot$ Behrokh Farahmand $^{2} \cdot$ Raziye Nazari $^{1} \cdot$ Amir Ghaemi $^{2} \mathbb{C}$
}

Accepted: 27 August 2021 / Published online: 12 September 2021

(c) The Author(s), under exclusive licence to Springer Nature B.V. 2021

\begin{abstract}
Influenza A viruses are among the most studied viruses, however no effective prevention against influenza infection has been developed. So, designing an effective vaccine against Influenza A virus is a critical issue in the field of medical biotechnology. For this reason, to combat this disease, we have designed a novel multi-epitope vaccine candidate based on the several conserved and potential linear B-cell and T-cell binding epitopes by using the in silico approach. This vaccine consists of an ER signal conserved sequence, the PADRE conserved epitope and two conserved epitopes of Influenza matrix protein 2. T-cell binding epitopes from Matrix protein 2 were predicted by in silico tools of epitope prediction. The selected epitopes were joined by flexible linkers and physicochemical properties, toxicity, and allergenecity were investigated. The designed vaccine was antigenic, immunogenic, and non-allergenic with suitable physicochemical properties and has higher solubility. The final multi-epitope construct was modeled, confirmed by different programs and the molecular interactions with immune receptors were considered. The molecular docking assay indicated the interactions with immune-stimulatory toll-like receptor 3 (TLR3) and major histocompatibility complex class I (MHCI). The HADDOCK and H DOCK servers were used to make docking analysis, respectively. The docking analysis indicated a strong and stable binding interaction between the vaccine construct with major histocompatibility complex (MHC) class I and toll-like receptor 3. Overall, the findings suggest that the current vaccine may be a promising vaccine to prevent Influenza infection.
\end{abstract}

Keywords Immunoinformatics · Multi-epitope vaccine · Toll-like receptor · In silico · Influenza type A · Vaccination · Matrix protein 2

\section{Introduction}

Influenza A viruses are still widely spread in nature and can infect a diversity of birds and mammals. Influenza A virus has always been a major reason of morbidity and mortality during history. Both the ability of the virus genome to change at a very high rate and its special gene mechanism often lead to the emergence of highly virulent strains that may potentially become the cause of a pandemic, or at least

Seyed Soheil Aghaei

soheilaghaee@yahoo.com; aghaei@qom-iau.ac.ir

Amir Ghaemi

ghaem_amir@yahoo.com; a_ghaemi@pasteur.ac.ir

1 Department of Microbiology, Qom Branch, Islamic Azad University, Qom, Iran

2 Department of Influenza and Other Respiratory Viruses, Pasteur Institute of Iran, 69, P.O.Box: 1316943551, Tehran, Iran some panic worldwide, for example the recent outbreak of swine-origin influenza A (H1N1) (Shao et al. 2017).

The swine-origin influenza A (H1N1) virus that appeared in 2009 and was first found in human beings in Mexico. The 2009 swine H1N1 flu pandemic-responsible for more than 17,000 deaths worldwide—originated in pigs from a very small region in central Mexico, a research team is reporting. The scientists say their findings represent the first time that the origin of an influenza pandemic virus has been determined in such detail (Shao et al. 2017).

Vaccination remains the most efficient strategies to control and prevent influenza infections in human and animal populations. Current vaccination approach against influenza virus consists of a live-attenuated or killed virus vaccine that are attention to be most widespread in the prospect influenza season. Thus, influenza vaccines have to be reformulated with the ever-changing influenza strains. In order to efficiently control and protect against pandemic outbreak, vaccine induced- immunity using conserved epitopes are 
protective and have been displayed to be an "universal" vaccine against various strains of the virus (Sabbaghi et al. 2021).

Influenza viruses belong to Orthomyxoviridae type A and type $\mathrm{B}$, which cause diseases in humans. Their genomes consists of eight single-stranded RNA segments that code for 10 different proteins, one nucleoprotein (NP), two external glycoproteins hemagglutinin (HA) and neuraminidase (NA), two nonstructural proteins (NS1 and NS2), two matrix proteins (M1 and M2) and three polymerase proteins (PA, $\mathrm{PB} 1$, and PB2). In view of the restrictions of usual influenza vaccines, researchers suggested an completely different approach derived from the greatly conserved extracellular domain of the viral M2 protein (M2e) (Ebrahimi and Tebianian 2011).

M2 is a structural protein, which serves a crucial function in the virus life cycle. The M2 is a type III membrane protein with 96 amino acid residues (Iwatsuki-Horimoto et al. 2006; Ebrahimi and Tebianian 2011). The N-terminal ectodomain (M2e) contains 23 residues; this sequence has stayed nearly unchanged since the first human influenza strain was isolated in 1933 (Meshcheryakova et al. 2009). As the M2e sequence is really conservative, many M2e vaccines have been designed and successfully experienced for value against a group of different influenza viruses in animal models (Kotomina et al. 2020). Here we focus on the development of universal influenza A vaccine candidates based on the conserved M2e epitopes. In addition, we have selected many conserved epitopes for vaccine development: ER (Ig j chain) (Määttänen et al. 2010), the PADRE Th epitope (Nezafat et al. 2014; Yarmarkovich et al. 2020). The selected epitopes were joined together using the appropriate linker to construct the multi-epitope vaccine.

In order to generate a stable immune response, it is important for the vaccine to interact with target immune cell receptors. For studying such interactions, molecular docking studies were performed with Toll-like receptors. Toll-like receptors (TLRs) have a central role in innate immunity as they detect conserved pathogen-associated molecular patterns (PAMPs) on a range of microbes, including viruses, leading to innate immune activation and adaptive immune response. Certain studies on Influenza A even indicated the noteworthy role of TLR 3 in generating protective responses against the virus. Immune responses are a crucial step to the pathophysiology of the Influenza virus-related disease, and initiation of immune response targeting TLR3 can trigger the anti-viral host defense mechanisms necessary for the elimination of the Influenza related infection (Astuti 2020). Therefore, molecular docking studies of the vaccine candidate with TLR3 was conducted.

Production of epitope-based vaccines by extremely conserved regions of Influenza virus proteins specially matrix protein 2 is considered as an important way to control the Influenza virus (Kumar et al. 2008; Astuti 2020). The improvement and creation of a vaccine are costly and will take many years to reach this goal, the different approaches were used to reduce the time and costs of this procedure (Suresh and Basu 2008). Reverse vaccinology is a new method that joins immunogenomics and bioinformatics to expand novel vaccines (Sarkar et al. 2020). This method has many benefits over usual vaccinology, it decreases the vaccine development time and costs. The computational programs enable to use a large immunological data; including antigen presentation and processing them to acquire sensitive analysis. Currently, in-silico vaccine design studies are facilitated via application of the applied bioinformatics tools; such as protein modeling programs, epitope prediction and protein-protein interaction analysis tools (Kumar et al. 2008). These vaccines are considered to be safer than traditional vaccines and focus on the most crucial antigenic elements of the pathogen to generate protective immunity (De Groot 2009). In this study, some specific B cell and T-cell epitopes from various proteins were chosen according to their antigenicity, stability, and length. The chosen epitopes were joined together by different linkers to construct the final potential multi-epitope construct, and different properties of the structures were predicted via in silico approaches.

\section{Method}

\section{Protein Sequence Retrieval}

The M2 protein (MATRIX PROTEIN 2) sequences of Influenza virus were retrieved from UniProtKB (www.uniprot. org/) Or from Influenza Virus Resource of National Center Biotechnology Information website (http://www.ncbi.nlm. nih.gov/genomes/FLU/FLU.html/) in FASTA format and the sequence was used for computational prediction. Then, analyses of the sequences were performed to study antigenicity, solvent-available areas, surface openness, litheness and MHC class-I binding sites (Hasan et al. 2013; Khan et al. 2015).

\section{Alignment of the Sequence}

M2 genes are one of the most conserved between Influenza serovars (with $99.1 \%$ similarities at the amino acid sequence level) (Ngamie 2020; Rosyda and Chaerul 2020). This was confirmed by Clustal omega, an algorithm of multiple amino acid sequence alignment available at (http://www.ebi.ac.uk/ Tools/msa/Clustalo). BLAST search was used to assemble the sequences of antigen reported for different serotypes (Zhang et al. 2015; Amimo et al. 2020). The resulting consensus sequence would characterize all of the virus's major serotypes. 


\section{Antigen Determinants}

To evaluate the physicochemical features of M2 antigen the ProtParam tool at (https://web.expasy.org/protparam/) (Tahir ul Qamar et al. 2020; Sharma, et al. 2021) was used. a number of factors given by the ProtParam tool, such as, molecular weight, theoretical pI, amino acid composition, atomic composition, extinction coefficient, estimated half-life, instability index, aliphatic index, and grand average of hydropathicity (GRAVY) were performed for this principle and self optimized prediction method with alignment (SOPMA) server at (https://npsa-prabi.ibcp.fr/cgi-bin/npsa_automat. pl?page=/NPSA/npsa_sopma.html) (Roy 2020) were used to obtain a prediction about the secondary structure of the chosen protein. Secondary structure calculation of a protein plans to forecast solvent convenience, transmembrane helices, globular areas, and coiled-coil regions. The purposes of this step were to conclude the protein's strength and task.

\section{Prediction of T-cell Epitopes}

Invariable forecasts of CTL (Cytotoxic T lymphocyte) epitopes are very critical for proposing consistent vaccine. For this reason, The T-cell epitopes of antigen (MHC I) was predicted, using the online prediction server Immune Epitope Database (IEDB) at (http://www.iedb.org/) (Vita et al. 2019). The binding affinity of the HLA haplotype to individual epitopes is the major factor related to the immune-dominance of CTL (Cytotoxic T lymphocyte) epitopes. According to allele frequency of different Iranian populations and among all HLA alleles, HLA-A*0201 is the most available ones in the world (Creary et al. 2019; Bahrami et al. 2020); Therefore, epitopes prediction was performed for this allele. The MHC I (HLA-A*0201)-restricted $\mathrm{T}$ cell epitopes were predicted by SYFPEITHI at (http:// www.syfpe ithi.de/) (Rashidian et al. 2020) and Bioinformatics and Molecular Analysis Section (BIMAS) at (https:// www-bimas.cit.nih.gov/molbio/hla_bind/) (Pan et al. 2017); Furthermore, MHCpred at (http://www.ddg-pharm fac.net/ $\mathrm{mhcpred} / \mathrm{MHCPred} /$ ) (Li et al. 2010).

\section{Design of the Multi-epitope Construct}

The purpose in increasing our $\mathrm{T}$ cell vaccine against influenza virus was initial to intend an immunogen using the conserved areas of influenza virus proteins like antigens and next to generate a recombinant vaccinia virus that can be applied as a wide-scale vaccine to stimulate specific immune response to influenza. For this aim, we created one kind of immunogen. Peptides were lined up together to satisfy one basic criteria: ER (Ig j chain) signal sequence. This signals the transportation of proteins into the ER where they are corrupted into peptides and loaded onto MHC class I molecules (Adler et al. 2017; Blackburn et al. 2017). The PADRE epitope has been shown to have a high MHCbinding affinity to a wide range of mouse and human MHC class II haplotypes and to induce Th responses restricted through class II of both species (Kissick et al. 2014; Yarmarkovich et al. 2020). Th1 peptide was separated by ARY extension since cytoplasmic Th1 epitope must gain access to secretory cavity to appear in the context of HLA class II molecules (Benveniste et al. 2015; Kuhn 2019). The flexible rich Alanine- aspartic acid linkers were used to join the selected epitopes. Besides linker we had added extra additions as Alanine/Arginine/Tyrosine (ARY) into siding area to enhance the peptide sympathy for TAP molecule (Salaun et al. 2020). the selected epitopes are fused together using proper amino acid linker from Linker Database at http:// www.ibi.vu.nl/programs/linkerdb. The flexible AD rich (one Alanine and one aspartic acid residues) linkers were used to join the selected epitopes. the multi-epitope were analysed with common on-line algorithms: NetCTL 1.2 (Kumar et al. 2020), nHLApred (Jahangiri et al. 2018),CTLPRED (Charoenkwan et al. 2020), TAPPRED (Bidmeshki et al. 2018),P-CLEAVAGE (Liu et al. 2019) and IEDB (Dhanda et al. 2019). This approach was followed for peptide arrangements of individual protein and then the most probable arrangements satisfying basic criteria was selected to be combined again in a longer polytope. the physicochemical features of the multi-epitope were calculated by ProtParam tool. After verification of the construct features, the multiepitope peptide was chemically synthesized in proper vector by biobasic Biological Engineering Co (CANADA).

\section{The Polytope Features Assessment}

The polytope proteins were then submitted in the VaxiJen v.2.0 server at (http://www.jenner.ac.uk/VaxiJen) with default parameters to isolate the antigenic proteins. To determine the antigenicity of the poly-epitope"virus" option was selected as a target organism. The accuracy of this server based on the target organisms (i.e. bacterial, viral, and tumor protein datasets). The ANTIGENpro at (http://scratch.prote omics.ics.uci.edu) was also used to predict the whole protein antigenicity (Mehmood et al. 2019). Protein allergenicity was predicted exploiting the AlgPred web server at (http:// www.imtech.res.in/raghava/algpred/) (Sharma et al. 2020) (accuracy with the threshold of 0.4).

\section{The Polytope Construct Secondary Structure}

We used SOPMA protein secondary structure prediction servers (Sharma et al. 2020). Additionally, for further verification, Jpred v.4 algorithm to predict the secondary structure of the polytope ( $>80.0 \%$ accuracy) (Khodabandelou and Nakib 2021). This server utilizes the hybrid method to 
predict the protein structure, which combines the alignment based and single sequence-based methods.

\section{The Polytope Construct Tertiary Structure}

The tertiary structure of the polytope protein was predicted using the 3DLigandSite server at (http://www.sbg.bio.ic. ac.uk/3dligandsite/) (Khodabandelou and Nakib 2021). The I-TASSER at (http://zhanglab.ccmb.med.umich.edu/ITASSER/download/) (Yang et al. 2015) was also applied to improve the accuracy of tertiary structure prediction. The quality of the 3D models was measured using the QMEAN and PROSA software at (https://swissmodel.expasy.org/ qmean/) (Pereira et al. 2019) and (https://prosa.services. came.sbg.ac.at/prosa.php) (Basak et al. 2020), respectively. The quality of the predicted structures was assessed by Rampage software at (http://mordred.bioc.cam.ac.uk/ rapper/ rampage.php) (Bahrami et al. 2020). Finally, the 3D models were visually analyzed by the PyMOL software (Pymol molecular graphics systemV. 1) (Bahrami et al. 2020; Gainza et al. 2020).

\section{Prediction of Cleavage Sites}

Netchop 3.1 at (http://www.cbs.dtu.dk/services/NetChop/) (Wang et al. 2017) and P-cleavage online servers were used to determine the proteasome cleavage sites of the polytope protein. Peptide binding affinity to TAP protein has been computed by TAPPred server at (http://crdd.osdd.net/ragha $\mathrm{va} /$ tappred/) (Keshtvarz et al. 2021).

\section{Molecular Docking}

The predicted multi-epitope was further tested by in silico docking simulation to find out whether or not this peptide will bind to the human leukocyte antigen (HLA) molecules and TLR3 molecule when it will be applied in vivo and The immunoreceptor-vaccine interaction is highly significant to elicit the immune response. Consequently, it was inexorable to perform molecular docking of the vaccine construct with immunological receptor molecule, TLR-3. Furthermore, the difficult among the ultimate vaccine and major histocompatibility complex (MHC-I) and TLR3 was calculated by molecular docking. To accomplish the simulation, a crystal structure of the HLA-A*02:01 molecule and TLR3, was retrieved from the Protein Data Bank (PDB) (Fatoba et al. 2021). Docking simulation for the predicted multi-epitope with prepared HLA-A*0201 and TLR3 were done by using HADDCK (High Ambiguity Driven protein-protein DOCKing) and HDOCK (Yan et al. 2020) which are informationdriven flexible docking approaches for the modeling of bio molecular complexes (Van Zundert et al. 2016). The Docked clusters were formed and based on the lowest HADDOCK and HDOCK score, and one cluster was identified. A representative structure from this cluster was subjected to molecular refinements for better positioning.

\section{Result}

\section{M2 Sequence Retrieval}

In current study, the protein sequence of the matrix protein 2 was retrieved from the UniProt database and then performed BLAST $p$. Based on previous articles, all the sequences were stored as a FASTA format for further analysis. From plenty of homologues, we have selected only 17 homologues having more than $60 \%$ identical sequences. MSA were performed and a phylogenetic tree was constructed. The findings from the MSA and phylogenetic data documented that the protein sequences have a closer relationship. The sequence under Gen Bank accession number of NP_040979.2 for M2 antigen was selected for the following analyses.

\section{Structure Analysis of Antigen Determinants}

The physiochemical properties for the matrix protein 2 antigen was determined in Table 1. The trans-membrane topology analysis of antigen showed that matrix protein 2 antigen could have transmembrane localization (Fig. 1).

\section{Epitope Prediction and Identification}

In this study, we have two categories of epitopes, which include antigenic and non-antigenic epitopes. The antigenic epitopes with highest scores which were confirmed with different software were selected as final epitopes

Table 1 Physicochemical parameters for matrix protein2 computed by ProtParam tool

\begin{tabular}{ll}
\hline Physicochemical properties & Matrix Protein2 \\
\hline Number of amino acids & 97 \\
Molecular weight & $11,044.70$ \\
Formula & $\mathrm{C}_{493} \mathrm{H}_{776} \mathrm{~N}_{134} \mathrm{O}_{144} \mathrm{~S}_{5}$ \\
Theoretical pI & 5.64 \\
Instability & 42.82 (unstable) \\
Estimated half-life (Escherichia coli, in vivo) & $30 \mathrm{~h}$ (mammalian \\
& reticulocytes \\
& in vitro) \\
& $>20 \mathrm{~h}$ (yeast, \\
& in vivo) \\
& $>10 \mathrm{~h}$ (Escherichia \\
& coli in vivo) \\
Aliphatic index & 93.51 \\
(GRAVY) & -0.207 \\
\hline
\end{tabular}


Fig. 1 A schema of transmembrane structure prediction of matrix protein 2 using TMHMM software

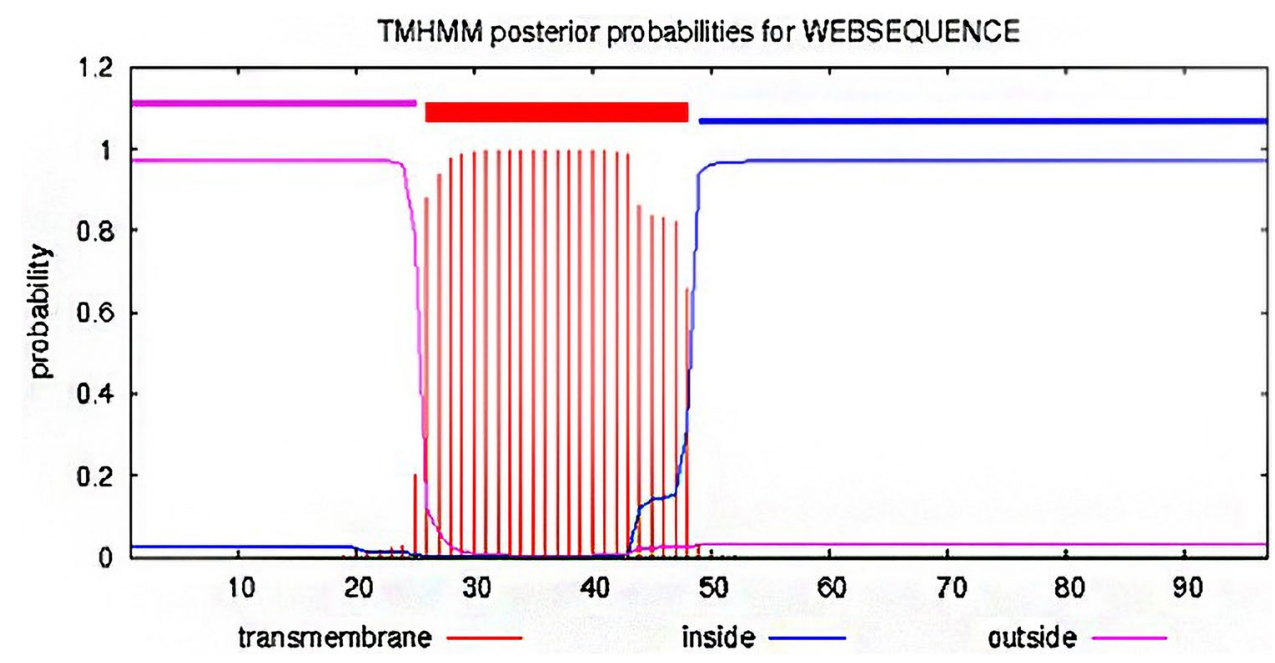

(Table 2). MHC I processing (this tool combines predictors of proteasomal processing, TAP transport and MHC binding to produce an overall score for each peptides potential of being a $\mathrm{T}$ cell epitope). Based on this overall score, six best epitopes were selected for further evaluation (Zhang et al. 2015; Amimo et al. 2020). The degree of similarity between the epitope and the target (i.e. given) sequence is elucidated by epitope conservancy. This property of epitope gives us the promise of its availability in a range of different strains. Hence for the analysis of the epitope conservancy, the web-based tool from IEDB analysis resources was used (http://www.iedb.org/) (Vita et al. 2019). Finally, two antigenic epitopes (SLLTEVETP (9 length) and EVETPIRN (9 length) with the most particular conserved and antigenic epitopes were chosen for further investigations.

Also, two non-antigenic epitopes ER signal sequence (MGMQVQIQSLFLLLLWVPGSRG) with 22 length and the PADRE (Th epitope) (AKFVAAWTLK) with 10 length were selected from previous articles (Adler et al. 2017; Blackburn et al. 2017).

This study focuses on predicting MHC-I binding epitopes and the prediction of MHC-II binding epitopes has not been done. It should be noted, there is only one MHC-II binding epitope in multi-epitope (the PADRE epitope
(AKFVAAWTLK)) structure that has been taken from previous articles (Kissick et al. 2014; Yarmarkovich et al. 2020).

\section{Design of the Polytope Construct and Polytope Arrangement Validation}

Peptides were lined up together to satisfy one basic criteria: ER (Ig jchain) signal sequence, the PADRE (Th epitope) and two high scores and more conservancy epitopes including the HLA-A*0201-restricted epitopes SLLTEVET and EVETPIRN (Valkenburg et al. 2016). The selected epitopes were fused together using proper amino acid linker from Linker Database at http://www.ibi.vu.nl/programs/linkerdb. The flexible AD (one alanine and one aspartic acid residues). Linkers have vital functions in the flexibility of the construct, protein folding, separating the functional domains and thus granting an overall stability to the protein. The selected epitopes of analyzed antigens were linked to each other using suitable linkers to build the final antigen. Besides linker we had added extra additions as Alanine/Arginine/ Tyrosine (ARY) into flanking area to increase the peptide affinity for TAP molecule (Seyed et al. 2014). The final construct of the polytope is consisted of 92 amino acid residues; ER region contributes 22 amino acids at the $\mathrm{N}$-terminal of
Table 2 Most potential 6 T-cell epitopes with total processing score and epitope conservancy result

\begin{tabular}{lllll}
\hline Protein & Start-end position & Epitope sequence & Conservancy & $\begin{array}{l}\text { MHC-I } \\
\text { processing } \\
\text { score }\end{array}$ \\
\hline Matrix protein 2 & $22-30$ & SSDPLAIAA & $30 \%$ & 0.95 \\
& $2-9$ & SLLTEVETP & $50 \%$ & 0.40 \\
& $6-13$ & EVETPIRN & $50 \%$ & 0.30 \\
& $3-11$ & LLTEVETPI & $40 \%$ & 0.94 \\
& $27-35$ & AIAANIIGI & $30 \%$ & 1.49 \\
& $83-91$ & AVDADDGHF & $40 \%$ & 2.35 \\
\hline
\end{tabular}


Fig. 2 Final arrangement of the polytope construct used for immunization

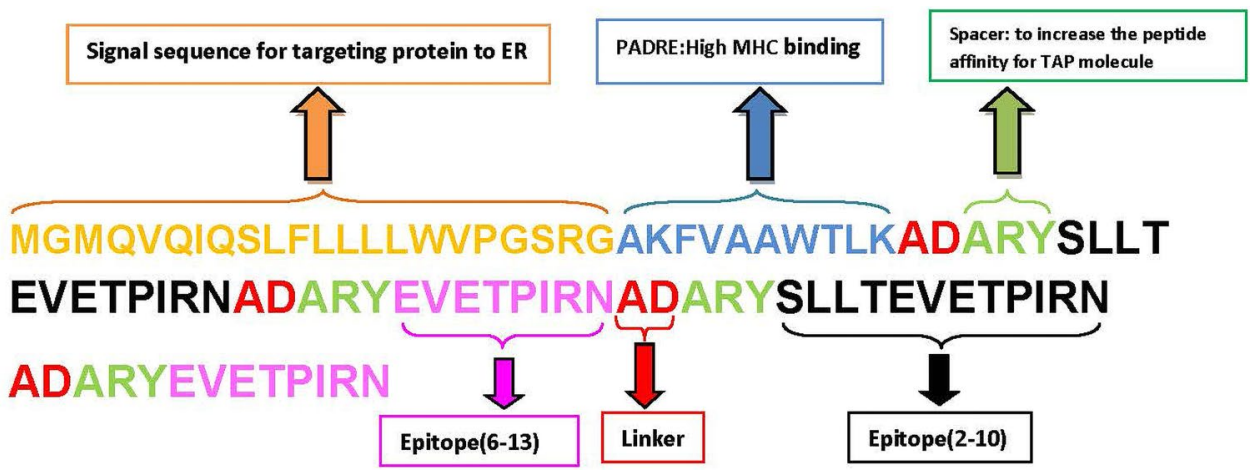

the construct $(1-22)$, the PADRE Th epitope (23-33) and 2 epitopes are derived from M2 protein of influenza virus, respectively (Fig. 2).

\section{The Polytope Features}

The physiochemical properties for each antigen were determined and summarized in Table 3. The isoelectric point (pI) and molecular weight (MW) of the polytope were computed to be 5.38 and $10,481.98 \mathrm{kDa}$, respectively. The protein halflife was estimated to be $3.5 \mathrm{~h}$ within mammalian reticulocytes (In vitro) and $>10 \mathrm{~h}$ within Escherichia coli (In vivo). The aliphatic index and instability index was defined to be 97.61 and 0.217 , respectively. These results indicate that the multi-epitope is a stable protein (Table 3 ).

\section{Antigenicity and Allergenicity}

The Vaxijen software is the first server for arrangementindependent forecast of defensive antigens; it was developed to allow antigen classification solely based on the physicochemical properties of proteins without recourse to sequence alignment. Based on this software the antigenicity of the multi-epitope construct with a threshold of 0.4 was predicted to be 0.3360 and $0.312 \%$ by ANTIGENpro. These results show that the multi-epitope is probably antigenic. The AllerTope results showed that the multi-epitope is probably non allergen. Moreover, based on the Algpred server results this protein is a non-allergen with the score of -1.366.

\section{The Multi-epitope Secondary Structure}

The computerized protein sequence was inserted in the available text box. The default output width is 70 . Meaning 70 amino acids are displayed in each line. The output width is changable. Regarding the parameters, options such as 'Number of conformational states', 'Similarity threshold' and 'Window width' are available. For the 'Number of conformational states', either '(3Helix, Sheet, Coil)' or '(4Helix, Sheet, Turn, Coil) can be selected. The former predicts the percentage of helix, sheet and coil structure while the latter predicts percentage of helix, coil, turn and sheet. Here we set the output width as 70; therefore 70 amino acids and corresponding predicted structures are visible in each line. The sequence length is also displayed in the output (92 amino acids in this case). The percentage of each structure is also included in this page. For instance, for Alpha helix it is $39.13 \%$. There are two graphs shown in the result page of SOPMA. The first page visualizes the prediction. The second contains score curves for all predicted states. It also shows the parameters such as window width, number of states etc. that are used for the prediction. It provides a link on the prediction result file which gives the result in a text format. There are links to find the intermediate result files also. The prediction of secondary structure by

Table 3 The prediction of binding affinity of TAP binder by TAPPred in poly-epitope protein

\begin{tabular}{|c|c|c|c|c|c|}
\hline Physicochemical Properties & ER & PADRE & SLLTEVETPIRN & EVETPIRN & POLYTOPE \\
\hline Number of amino acids & $2 \mathrm{aa}$ & $10 \mathrm{aa}$ & $12 \mathrm{aa}$ & $8 \mathrm{aa}$ & $92 \mathrm{aa}$ \\
\hline Molecular Weight & 2474.02 & 1134.39 & 1371.55 & 957.05 & $10,481.98$ \\
\hline Theoretical pI & 9.50 & 10.00 & 4.53 & 4.53 & 5.38 \\
\hline Instability & 43.22(unstable) & 14.52(stable) & 35.50(unstable) & 24.18(stable) & 15.41 (stable) \\
\hline $\begin{array}{l}\text { Estimated half-life (Escherichia } \\
\text { coli, in vivo) }\end{array}$ & $>10 \mathrm{~h}$ & $>10 \mathrm{~h}$ & $>10 \mathrm{~h}$ & $>10 \mathrm{~h}$ & $>10 \mathrm{~h}$ \\
\hline Aliphatic index & 132.73 & 98.00 & 121.67 & 85.00 & 97.61 \\
\hline (GRAVY) & 0.827 & 0.680 & -0.208 & -1.075 & -0.217 \\
\hline
\end{tabular}


Fig. 3 A The predicted Secondary structure plot of multiepitope construct secondary using SOPMA software. H: Alpha helix, E: Extended strand, T: Beta-turn and C: Random coil. B The graphical representation of secondary elements in multi-epitope construct (blue: alpha helix, red: extended strand, green: beta turn, yellow: random coil) (Color figure online)
A

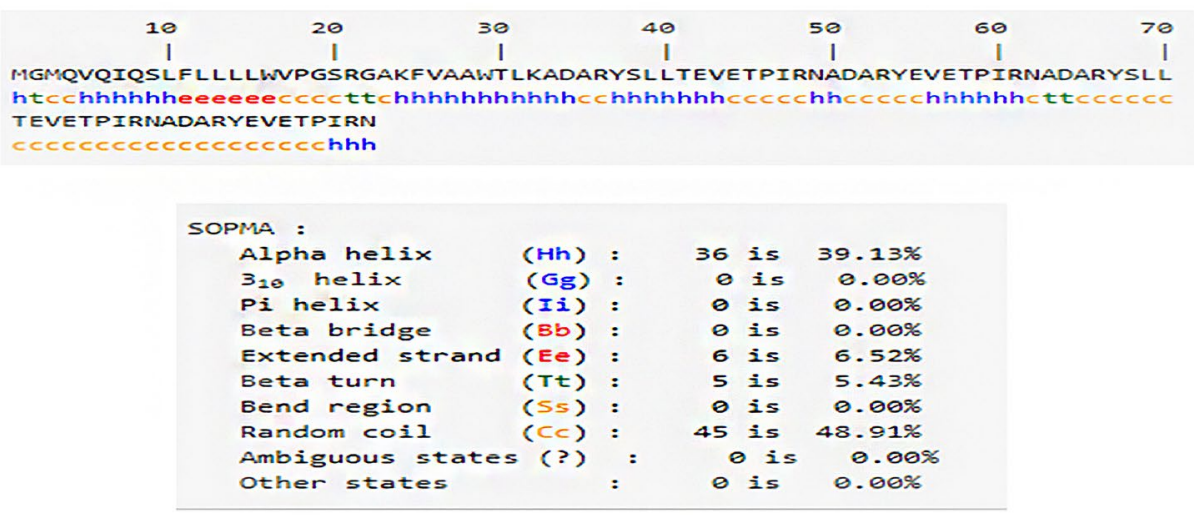

B
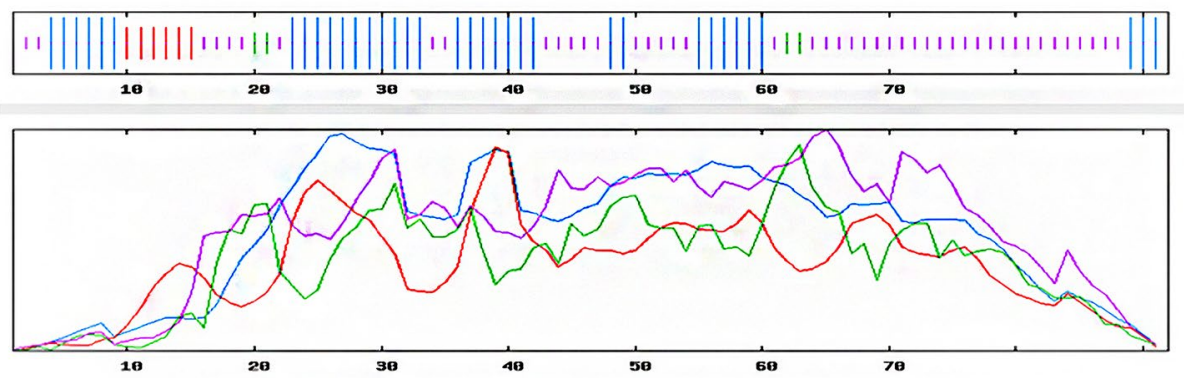

SOPMA indicated that the multi-epitope consists of $39.13 \%$ $\alpha$-helix (H), 6.52\% extended strand (E), 5.43\% beta turn (T) and $48.91 \%$ random coil (C) elements (Fig. 3). The high ratio of random coils and extended strands in the structure of multiepitope indicates that the designed protein is probably capable of forming antigenic epitopes.

\section{Multi-epitope Tertiary Structure and Refinement}

The Phyre and I-TASSER servers have returned one and five 3D models of the designed protein, respectively. Between the five models that were presented by I-TASSER, a model with the highest $\mathrm{C}$-score (-0.77) was selected as the best model (Fig. 4). It is worth noting the C-score is usually within the range of (McMahon 2014; McLauchlan 2000), the higher $\mathrm{C}$-scores are associated with high confidence and vice versa. The higher quality of the selected model was confirmed by QMEAN results. The ProSA result indicated that the selected model did not appear within the range of native proteins of similar size and needs to be refined. The selected primary model was refined and energy minimized. The model refinement and energy minimization run have improved the quality of the selected 3D model.

\section{Multi-epitope Tertiary Structure Validation}

To validate the quality of the refined model, two online validation methods like Ramachandran plot (https://servicesn. mbi.ucla.edu/PROCH
ECK/) (Laskowski et al. 2012) and ProSA (https://prosa. services.came.sbg.ac.at/prosa.php) (Wiederstein and Sippl 2007) were used. Ramachandran plot assessment showed that $84.6 \%$ of the structure was under favored region, $13.3 \%$ was under the allowed region, and $2.1 \%$ was observed under the disallowed region, signaling a high quality of the predicted vaccine structure (Fig. 5). Additionally, Pros A-web showed a Z-score of -3.85 , which lies inside the range of acceptable scores and Z-score was required prior to studying molecular interactions (Haghighi et al. 2020; Haghighi and Moradi 2020). The obtained results suggest that the refinement run have relocated most of the amino acids within the allowed area.

\section{Docking Simulation Results}

Molecular docking studies can reveal the binding interactions between modeled protein and receptor molecules. For this purpose, we submitted the refined vaccine model as ligand and HLA-A*02:01 and TLR3 proteins as immunological receptors into the HADDOCK output (Vangone et al. 2017), for molecular docking. The HADDOCK output (Vangone et al. 2017) consisted of several models, among which the 10 highest clusters were selected. According to HADDOCK, the most valid cluster is the top one; however, this ranking also depends on its $\mathrm{Z}$-score: the more negative the $\mathrm{Z}$-score the more reliable the cluster. The top cluster is the most reliable according to HADDOCK. Its Z-score indicates how many standard 
Fig. 4 Representation of the modeled structure using (The PYMOL Molecular Graphics System). A structure of predicted multi-epitope. B structure of HLA-A*02:01. C structure of toll like receptor3

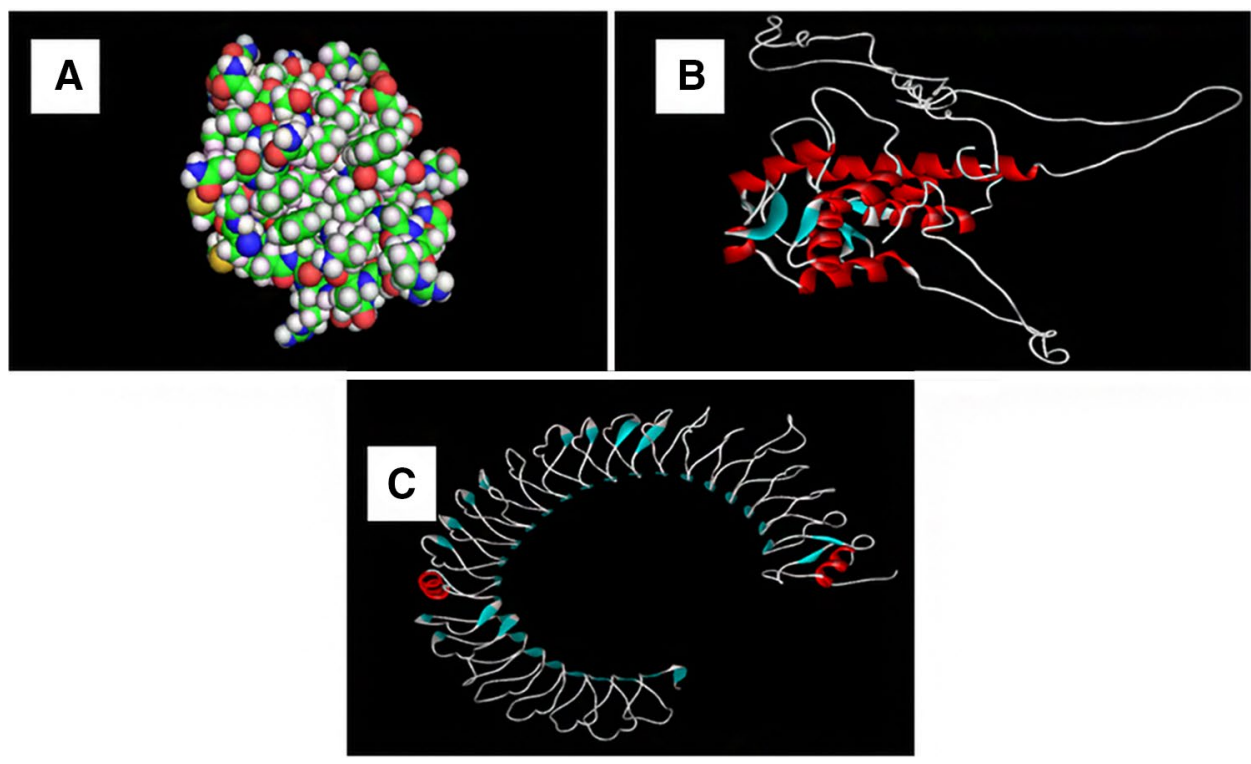

deviations from the average this cluster is located in terms of score (the lower the z-score is, the better). The docking analysis showed good interaction between the vaccine construct and HLA-A*02:01 with -1.9 Z-score (Fig. 6). The TLR3 receptor (PDB ID- $2 \mathrm{~A} 0 \mathrm{Z}$ ) was selected and downloaded from the PDB server (Sussman et al. 1998). Initially, the receptor was prepared by separating the attached ligand from the protein, followed by the removal of waters and other chemicals. All these processes were performed in PyMOL v2.3.4 software (DeLano 2002). Binding interactions and residues involved in the interacting plane were analyzed with Discovery Studio 2017. The docking between the vaccine (ligand) and TLR3 (receptor) was performed to anticipate their binding affinity and interactions. In doing so, the HDOCK server provided 30 docked complexes with different poses. Among them, we selected the complex with the least energy score and binding pose with functional interactions. Thus, model 3 of TLR-3 - vaccine docked having complex lowest energy of $-323.73 \mathrm{kcal} / \mathrm{mol}$, were selected for further analysis (Fig. 7).

\section{Cleavage Site and TAPPRED}

P-cleavage (server for identification of protesomal cleavage sites in a protein sequence) and Net Chop servers have been used for selected cleavage site. The servers have predicted 92 cleavage sites for the multi-epitope protein (data not shown). The proteasome complex consists of enzymes that cut the peptide bonds, changing the proteins into peptides. The peptide molecules from proteasome cleavage connect with class-1 MHC molecules and the peptide-MHC molecules are then moved to the cell membrane where they are presented to $\mathrm{T}$ helper cell.
The multi-epitope TAP binding affinity results, predicted by TAPPred (The server that prediction of TAP binding peptides for understanding of peptide internalization to ER) are listed in Table 4. In this case, the total score was predicted and the higher the score the higher the efficiency of all these processes/a higher score meant a higher efficiency for all these processes (Fig. 7).

\section{Discussion}

Influenza remains a serious respiratory disease in spite of the availability of anti-virals and inactivated trivalent vaccines, which are effective for most recipients. The key challenges for creating vaccines against influenza A virus are antigenic drift and antigenic shift. Preventive effects of present influenza virus vaccines are quite specific to particular strains of influenza virus and are based generally on antibody responses to surface antigens of the virus hemagglutinin and neuraminidase (Nachbagauer et al. 2019). Thus, influenza virus vaccines are not capable to afford protection from a different scale of viral strains. Therefore, to evade eruptions of epidemics among human populations, modern vaccine needs the growth of new universal and multi-epitope vaccines that are able to offer protection from a large variety of influenza A virus strains and would not need to be updated every year.

Multi-epitope vaccines offer a viable alternative since they can elicit potent immune responses without causing undesirable allergic reactions and have been already used against a variety of Influenza strains successfully (Rakib et al. 2020). These vaccines are a group of recombinant products with high specificity, safety, stability and lowcost of production (Chan et al. 2020). Therefore, any study 
Fig. 5 Predicted 3D structure and validation of multi-epitope vaccine construct. A 3D structure of vaccine construct. B Ramachandran plot showed that $84.6 \%$ of the proposed vaccine construct residues were present in favored regions, $13.3 \%$ in allowed regions while only $2.1 \%$ in disallowed regions. $\mathbf{C}$ PROSA validation of $3 \mathrm{D}$ structure showing $\mathrm{Z}$-score $(-3.85)$. The z-score indicates overall model quality
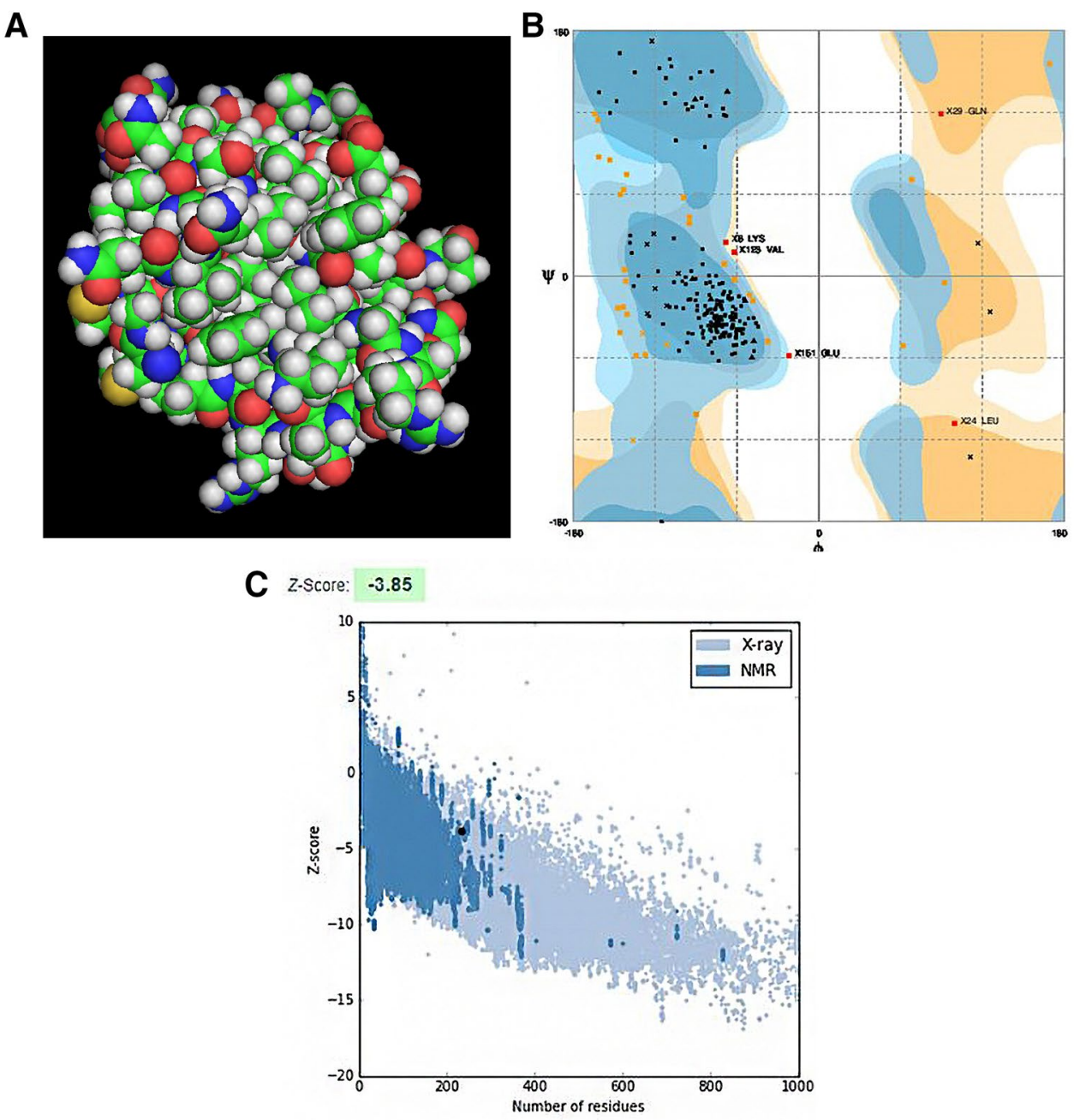

that could help to improve the quality of these agents is important. Since the laboratory-based works require application of a considerable amount of consumables, materials, human workforce, and sacrifice of animals; using computer-based methods could help to reduce the burden from laboratory, and decrease the costs of errors by using insilico methods. Immunoinformatics or computational immunology is a branch of bioinformatics that includes investigations such as B- and T-cell epitope mapping, along with many other aspects including toxicity, and allergenicity (Raghuvanshi et al. 2020). The potential of insilico designed vaccines for Influenza has also been confirmed by many in vivo and in vitro studies, for example, a study by Rodrigueza et al. reported a novel epitope-based vaccine candidate with protective efficacy against Influenza A in a mouse model, and the output confirmed efficacy of the designed peptide against the virus (Onyango et al. 2012). Generation of novel vaccines that can overcome antigenic diversity and inherent low immunogenicity of vaccines against Influenza A virus is critical for pandemic preparation. In this regard, in silico tools were utilized at the current research. Moreover, application of the graphic approaches for studying the biological and medical systems could present an intuitive knowledge to help analyzing the complicated molecular interactions (Chou 2020).

Our purpose was to design an epitope vaccine by targeting the Matrix protein 2 which is a viral ion channel protein that mediates viral uncoating and is the target protein for the therapeutic drug amantadine, and its methyl derivative rimantadine (Jalily et al. 2020). The M2 protein can be segregated into three parts: the extracellular $\mathrm{N}$-terminal domain (M2e, locations 2-24), the trans membrane (TM) domain (locations 25-46) and the intracellular C-terminal domain (positions 47-97) (72). The extracellular part of the M2 protein is remarkably conserved, as shown by alignment of the sequences of M2 proteins, isolated from different human strains of influenza A virus (De Filette et al. 2006). The high degree of conservation of M2 makes it capable of stimulating both humoral and cellular immune responses (Kordon et al. 2018). The ectodomain of influenza A virus M2 protein (M2e) is made of 24 amino acids (Kolpe et al. 
Cluster 3

$\begin{array}{ll}\text { HADDOCK score } & -152.6+/-9.5 \\ \text { Cluster size } & 11 \\ \text { RMSD from the overall lowest-energy structure } & 0.9+/-0.6 \\ \text { Van der Waals energy } & -83.3+/-7.0 \\ \text { Electrostatic energy } & -370.7+/-50.9 \\ \text { Desolvation energy } & -42.0+/-6.5 \\ \text { Restraints violation energy } & 467.2+/-63.85 \\ \text { Buried Surface Area } & 2844.5+/-111.0 \\ \text { Z-Score } & -1.9\end{array}$

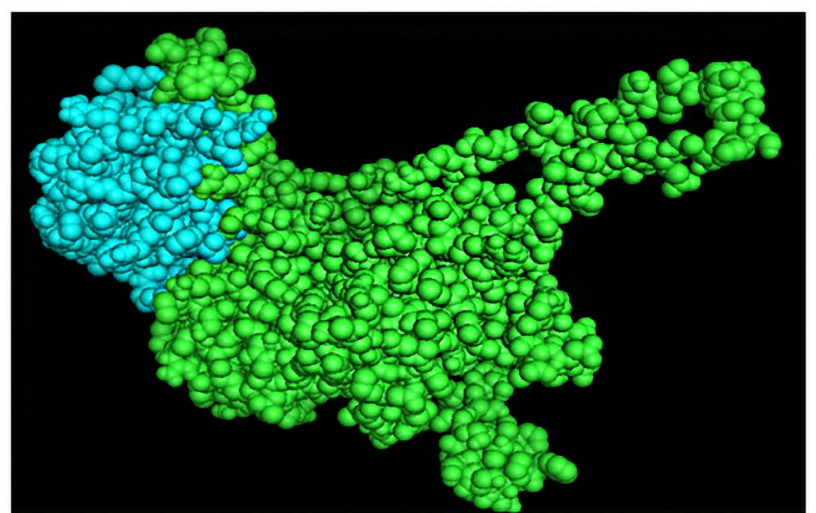

Fig. 6 HLA-A*02:01 is shown in the green color, while the vaccine construct is shown in the blue color and the best result of HADDOCK, respectively (Color figure online)

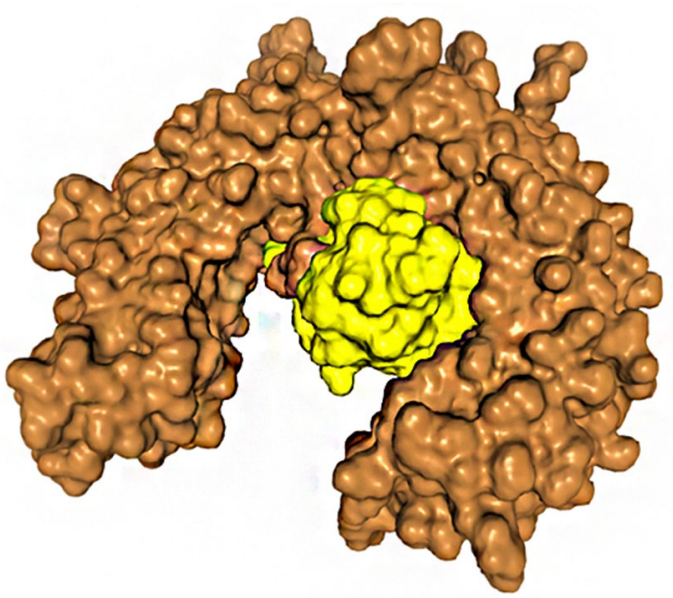

Fig. 7 Visualization of interacting residues of docked complex. Toll like receptor 3 is shown in the brown color, the vaccine construct is shown in the yellow color, with lowest energy weighted score -323.73 (Color figure online)

Table 4 The prediction of binding affinity of TAP binder by TAPPred in poly-epitope protein

\begin{tabular}{lllll}
\hline Peptide Rank & Start Position & Sequence & Score & \\
\hline 1 & 23 & AKFVAAWTL & 9.878 & High \\
2 & 35 & ARYSLLTEV & 8.088 & High \\
3 & 65 & AKFVAAWTL & 8.088 & High \\
4 & 9 & SLFLLLLWV & 7.110 & High \\
5 & 31 & LKADARYSL & 7.006 & High \\
6 & 52 & ARYEVETPI & 6.433 & High \\
7 & 82 & ARYEVETPI & 6.433 & High \\
8 & 61 & RNADARYSL & 6.160 & High \\
9 & 10 & LFLLLLWVP & 6.149 & High \\
10 & 62 & NADARYSLL & 6.032 & High \\
\hline
\end{tabular}

2017; Mezhenskaya et al. 2019). Until recently, there were several universal flu vaccine candidates that focused on the highly conserved M2e protein. A area close to the beginning of M2e which is general to both M1 and M2 proteins, was exposed to be the highly conserved epitope, SLLTEVET. Definitely, monoclonal antibodies (mAb) against this epitope strongly reduced the replication of influenza A virus $\mathrm{H} 1$ and H3 subtypes in MDCK cells (Yamayoshi et al. 2017). This particular area of $\mathrm{M} 2 \mathrm{e}$ is still believed to characterize a probable epitope to be contained in a generally protective influenza vaccine. Furthermore, it has been demonstrated that the epitope EVETPIRN (aa6-13) on M2e could be responsible for the stimulation of the protective immunity (Zhou et al. 2012).

At the current research, we aimed to design a potential multi-epitope vaccine candidate that contain the conserved epitopes of Matrix protein 2, ER (Ig jchain) the signal sequence and the 'universal' Th epitope (PADRE). we used Repetition of M2 selective epitopes in construct for increasing immune system stimulation (de Matos et al. 2020). The coupling between matrix protein 2 epitopes that is mentioned above, invokes a desired increase in effectiveness of the protein (Kirsteina et al. 2020). ER signal sequence directs the ribosome to the ER membrane and initiates transport of the growing poly-peptide across the ER membrane (Serwold et al. 2002). This signal targets processing of proteins to the ER where they are corrupted into peptides and loaded onto MHC class I molecules. The 'universal' Th epitope, Pan-DR epitope (PADRE) has been shown to have a high MHC-binding affinity to a wide range of mouse and human MHC class II haplotypes (Rosa et al. 2010).

The multi-epitope construct was designed based on the conserved epitopes of different proteins, attached together using different linkers, and was subjected to different bioinformatics assays. 
It is remarkable that linkers could have an impact on the role and arrangement of multi-epitope vaccines. In this approach, the last multi-epitope construct has been designed including the flexible linkers between the selected epitopes. Flexible linkers are generally wealthy in small or polar amino acids such as Alanine and Aspartic acid to offer good flexibility and solubility (Chen et al. 2013).

The physicochemical properties of the proteins play pivotal roles in various biological designs. The computed pI, instability index, aliphatic index, GRAVY index values reveal stability, thermostability, hydrophobicity of the multiepitope protein respectively. These properties showed that hydrophilic residues could be placed on the protein surface and they are suitable for ligand binding.

The vaccine structure has a half-life of 30,20 , and $10 \mathrm{~h}$ in mammalian, yeast, and E. coli, respectively, therefore the vaccine is exposed to the immune system for a relatively long time and causes more immune responses (NikolichŽugich 2018). The multi-epitope vaccine's instability index was calculated at 15.41 , and as it was less than 40 , it was considered to be a stable protein (Singh et al. 2020). The calculated aliphatic index and the GRAVY were 97.61 and -0.217 , respectively. The higher aliphatic index value suggests greater thermostability and the negative GRAVY value shows the vaccine's hydrophilic nature therefore it can show strong interaction with water molecules (Sanami et al. 2020). Results also suggested that the vaccine was soluble, non allergenic, and non-toxic.

The secondary structure of protein is predicted using SOPMA (Self- Optimized Prediction Method with Alignment). This tool evaluates properties such as solvent accessibility, globular regions, transmembrane helices, random coil, bend region and coiled-coil region. The secondary structure analysis revealed higher random coil content in the construct. Random coils play an important role in the high flexibility of proteins. The high coil structural percentage of the designed multi-epitope (48.91\%) was due to the high content of Alanine-Aspartic acid linker sequences. The role of Alanine-aspartic-rich residue in protein flexibility has been reported previously (Elfiky 2020).

Information regarding the three-dimensional (3D) structures of proteins and their complexes with their potential ligands are of critical importance for designing novel therapeutic agents; such as multi-epitope vaccines. Application of the in silico methods for prediction of 3D structure of the protein has been proven an efficient method by using bioinformatics algorithms (Alkema et al. 2016). The 3D structure of multi-epitope construct was modeled by I-TASSER and Structure of this construct was confirmed by different programs, considering different aspects, such as Ramachandran plot, GalaxyRefine server,RAMPAGE server and ProSAweb. In the validation test of 3D structure, we found a good number of Z-score (-3.85) and the superior features of most favored, accepted and disallowed regions for the Ramachandran plot. HADDOCK server conducted the docking process between the multi-epitope vaccine with HLA-A*02:01 and TLR3. the value of lowest energy and Z-score indicated that the interaction of the vaccine with the HLA-A*02:01 and TLR3. The score of lowest energy and Z-score showed good interaction between them.

Overall, this study provided a potential multi-epitope peptide vaccine candidate against Influenza A virus; based on the different conserved epitopes. The limitations of currently in market seasonal Influenza vaccines and the persistent threat of future pandemics have made it necessary for novel vaccine design. This designed construct could cover many subtypes of the virus and serve as wide rage protection against this seasonal disease. In this study, a series of computational approaches led to the discovery of potential $\mathrm{T}$ - and B-cell epitopes that eventually embroidered into a multi-epitope vaccine. These epitopes were chosen based on their antigenicity and stability. Importantly, it was able to bind with the immune receptor TLR3 and MHC class I strongly as well as to elicit strong immune response upon Influenza infection. The results provided by the current in silico study showed that the multi-epitope construct can provide promising outcomes against Influenza A virus and could be devoted for future in vitro, and in vivo studies.

Acknowledgements The authors would like to acknowledgement Pasteur institute of Iran for the financial support.

Funding This work was supported by Pasteur Institute of Iran (Grant 1137).

Data Availability The datasets used and analysed during the current study are available from the corresponding author on reasonable request.

\section{Declarations}

Conflict of interest Authors declare there is no conflict of interest.

Research Involving Human and/or Animal Participants This article does not contain any studies with human participants or animals performed by any of the authors.

\section{References}

Adler LN, Jiang W, Bhamidipati K, Millican M, Macaubas C, Hung S-C, Mellins ED (2017) The other function: class II-restricted antigen presentation by B cells. Front Immunol 8:319

Alkema L, Chou D, Hogan D, Zhang S, Moller A-B, Gemmill A, Fat DM, Boerma T, Temmerman M, Mathers C (2016) Global, regional, and national levels and trends in maternal mortality between 1990 and 2015, with scenario-based projections to 2030: 
a systematic analysis by the UN Maternal Mortality Estimation Inter-Agency Group. Lancet 387(10017):462-474

Amimo JO, Machuka EM, Abworo EO, Vlasova AN, Pelle R (2020) Whole genome sequence analysis of porcine astroviruses reveals novel genetically diverse strains circulating in East African Smallholder pig farms. Viruses 12(11):1262

Astuti I (2020) Severe Acute Respiratory Syndrome Coronavirus 2 (SARS-CoV-2): an overview of viral structure and host response. Diabetes Metab Syndr 14(4):407-412

Bahrami AA, Bandehpour M, Khalesi B, Kazemi B (2020) Computational design and analysis of a poly-epitope fusion protein: a new vaccine candidate for Hepatitis and Poliovirus. Int J Pept Res Ther 26(1):389-403

Basak N, Krishnan V, Pandey V, Punjabi M, Hada A, Marathe A, Jolly M, Palaka BK, Ampasala DR, Sachdev A (2020) Expression profiling and in silico homology modeling of Inositol penta kis phosphate 2-kinase, a potential candidate gene for low phytate trait in soybean. 3 Biotech 10:1-21

Benveniste O, Stenzel W, Hilton-Jones D, Sandri M, Boyer O, van Engelen BG (2015) Amyloid deposits and inflammatory infiltrates in sporadic inclusion body myositis: the inflammatory egg comes before the degenerative chicken. Acta Neuropathol 129(5):611-624

Bidmeshki, T., A. M. Ahadi and H. Ayat (2018). "A New Design and Epitopes Analysis for Recombinant Vaccine Against Salmonella typhi by In silico Analysis."

Blackburn, T. E., T. Santiago and P. D. Burrows (2017). FCRLA-a resident endoplasmic reticulum protein that associates with multiple immunoglobulin isotypes in B lineage cells. IgM and Its Receptors and Binding Proteins: 47-65.

Chan Y, Jazayeri SD, Ramanathan B, Poh CL (2020) Enhancement of tetravalent immune responses to highly conserved epitopes of a dengue peptide vaccine conjugated to polystyrene nanoparticles. Vaccines 8(3):417

Charoenkwan P, Nantasenamat C, Hasan MM, Shoombuatong W (2020) iTTCA-Hybrid: Improved and robust identification of tumor $\mathrm{T}$ cell antigens by utilizing hybrid feature representation. Anal Biochem 599: 113747

Chen X, Zaro JL, Shen W-C (2013) Fusion protein linkers: property, design and functionality. Adv Drug Deliv Rev 65(10):1357-1369

Chou K-C (2020) Proposing pseudo amino acid components is an important milestone for proteome and genome analyses. Int J Pept Res Ther 26(2):1085-1098

Creary LE, Gangavarapu S, Mallempati KC, Montero-Martín G, Caillier SJ, Santaniello A, Hollenbach JA, Oksenberg JR, Fernández-Viña MA (2019) Next-generation sequencing reveals new information about HLA allele and haplotype diversity in a large European American population. Hum Immunol 80(10):807-822

De Filette M, Fiers W, Martens W, Birkett A, Ramne A, Löwenadler B, Lycke N, Jou WM, Saelens X (2006) Improved design and intranasal delivery of an M2e-based human influenza A vaccine. Vaccine 24(44-46):6597-6601

DeLano WL (2002) Pymol: an open-source molecular graphics tool. CCP4 Newsl Prot Crystallogr 40(1):82-92

De Groot GJ (2009) The sixties unplugged: a kaleidoscopic history of a disorderly decade. Harvard University Press

De Matos AL, Franco LS, McFadden G (2020) Oncolytic viruses and the immune system: the dynamic duo. Mol Ther Method Clin Dev 17:349-358

Dhanda SK, Mahajan S, Paul S, Yan Z, Kim H, Jespersen MC, Jurtz V, Andreatta M, Greenbaum JA, Marcatili P (2019) IEDB-AR: immune epitope database-analysis resource in 2019. Nucleic Acids Res 47(W1):W502-W506

Ebrahimi SM, Tebianian M (2011) Influenza A viruses: why focusing on M2e-based universal vaccines. Virus Genes 42(1):1-8
Elfiky AA (2020) Anti-HCV, nucleotide inhibitors, repurposing against COVID-19. Life Sci 248: 117477

Fatoba AJ, Maharaj L, Adeleke VT, Okpeku M, Adeniyi AA, Adeleke MA (2021) Immunoinformatics prediction of overlapping CD8+ T-cell, IFN- $\gamma$ and IL-4 inducer CD4+ T-cell and linear B-cell epitopes based vaccines against COVID-19 (SARS-CoV-2). Vaccine 39(7):1111-1121

Gainza P, Sverrisson F, Monti F, Rodola E, Boscaini D, Bronstein M, Correia B (2020) Deciphering interaction fingerprints from protein molecular surfaces using geometric deep learning. Nat Methods 17(2):184-192

Haghighi O, Davaeifar S, Zahiri HS, Maleki H, Noghabi KA (2020) Homology modeling and molecular docking studies of glutamate dehydrogenase (GDH) from cyanobacterium Synechocystis sp. PCC 6803. Int J Pept Res Th 26(2):783-793

Haghighi O, Moradi M (2020) In silico study of the structure and ligand interactions of alcohol dehydrogenase from Cyanobacterium Synechocystis sp. PCC 6803 as a key enzyme for biofuel production. Appl Biochem Biotech 192(4):1346-1367

Hasan A, Hossain M, Alam J (2013) A computational assay to design an epitope-based Peptide vaccine against Saint Louis encephalitis virus. Bioinform Biol Insights 7: BBI. S13402.

Iwatsuki-Horimoto K, Horimoto T, Noda T, Kiso M, Maeda J, Watanabe S, Muramoto Y, Fujii K, Kawaoka Y (2006) The cytoplasmic tail of the influenza A virus M2 protein plays a role in viral assembly. J Virol 80(11):5233-5240

Jahangiri A, Amani J, Halabian R (2018) In silico analyses of staphylococcal enterotoxin B as a DNA vaccine for cancer therapy. Int J Pept Res Ther 24(1):131-142

Jalily PH, Duncan MC, Fedida D, Wang J, Tietjen I (2020) Put a cork in it: Plugging the M2 viral ion channel to sink influenza. Antiviral Res 178:104780

Keshtvarz M, Salimian J, Amani J, Douraghi M, Rezaie E (2021) In silico analysis of STX2a-PE15-P4A8 chimeric protein as a novel immunotoxin for cancer therapy. Silico Pharmacol $9(1): 1-12$

Khan M, Hossain M, Rakib-Uz-Zaman S, Morshed M (2015) Epitope-based peptide vaccine design and target site depiction against Ebola viruses: an immunoinformatics study. Scand J Immunol 82(1):25-34

Khodabandelou G, Nakib A (2021) H-polytope decomposition-based algorithm for continuous optimization. Inf Sci 558:50-75

Kirsteina A, Akopjana I, Bogans J, Lieknina I, Jansons J, Skrastina D, Kazaka T, Tars K, Isakova-Sivak I, Mezhenskaya D (2020) Construction and immunogenicity of a novel multivalent vaccine prototype based on conserved Influenza virus antigens. Vaccines 8(2): 197

Kissick HT, Sanda MG, Dunn LK, Arredouani MS (2014) Immunization with a peptide containing MHC class I and II epitopes derived from the tumor antigen SIM2 induces an effective CD4 and CD8 T-cell response. PLoS ONE 9(4): e93231

Kolpe A, Schepens B, Fiers W, Saelens X (2017) M2-based influenza vaccines: recent advances and clinical potential. Expert Rev Vaccines 16(2):123-136

Kordon AO, Karsi A, Pinchuk L (2018) Innate immune responses in fish: antigen presenting cells and professional phagocytes. Turk J Fish Aquat Sci 18(9):1123-1139

Kotomina T, Isakova-Sivak I, Kim K-H, Park BR, Jung Y-J, Lee Y, Mezhenskaya D, Matyushenko V, Kang S-M, Rudenko L (2020) Generation and characterization of universal live-attenuated influenza vaccine candidates containing multiple M2e epitopes. Vaccines 8(4):648

Kuhn KA (2019) Overview of the immune response. Inflamm Autoimmunity Rheumatology Secrets E-Book: 26 
Kumar A, Rotter S, Aertsen A (2008) Conditions for propagating synchronous spiking and asynchronous firing rates in a cortical network model. J Neurosci 28(20):5268-5280

Kumar S, Maurya VK, Prasad AK, Bhatt ML, Saxena SK (2020) Structural, glycosylation and antigenic variation between 2019 novel coronavirus (2019-nCoV) and SARS coronavirus (SARSCoV). Virusdisease 31(1):13-21

Laskowski R, MacArthur M, Thornton J (2012) Other validation tools

Li Z, Zhang M, Hu H, Liu S, Lu Z (2010) On predicting the T cell and B cell epitopes of platelet membrane glycoprotein II b/III a antibody from human and mice. J Biomed Eng 27(5): $1146-1151$.

Liu T, Zhu J, Sun X, Cheng L, Wu L (2019) I2/TBHP mediated divergent C (sp2)-P cleavage of allenylphosphine oxides: substituentcontrolled regioselectivity. Adv Synth Catal 361(15):3532-3537

Määttänen P, Gehring K, Bergeron JJ, Thomas DY (2010) Protein quality control in the ER: the recognition of misfolded proteins. Semin Cell Dev Biol

McLauchlan J (2000) Properties of the hepatitis C virus core protein: a structural protein that modulates cellular processes. J Viral Hepatitis 7(1):2-14

McMahon BJ (2014) Chronic hepatitis B virus infection. Med Clin 98(1):39-54

Mehmood A, Kaushik AC, Wei DQ (2019) Prediction and validation of potent peptides against herpes simplex virus type 1 via immunoinformatic and systems biology approach. Chem Biol Drug Des 94(5):1868-1883

Meshcheryakova YA, Eldarov M, Migunov A, Stepanova L, Repko I, Kiselev C, Lomonossoff G, Skryabin K (2009) Cowpea mosaic virus chimeric particles bearing the ectodomain of matrix protein 2 (M2E) of the influenza A virus: production and characterization. Mol Biol 43(4):685-694

Mezhenskaya D, Isakova-Sivak I, Rudenko L (2019) M2e-based universal influenza vaccines: a historical overview and new approaches to development. J Biomed Sci 26(1):1-15

Nachbagauer R, Salaun B, Stadlbauer D, Behzadi MA, Friel D, Rajabhathor A, Choi A, Albrecht RA, Debois M, García-Sastre A (2019) Pandemic influenza virus vaccines boost hemagglutinin stalk-specific antibody responses in primed adult and pediatric cohorts. NPJ Vaccines 4(1):1-12

Nezafat N, Ghasemi Y, Javadi G, Khoshnoud MJ, Omidinia E (2014) A novel multi-epitope peptide vaccine against cancer: an in silico approach. J Theor Biol 349:121-134

Ngamie WM (2020) Genetic detection and characterization of Influenza viruses in selected wildlife and humans at high-risk interface in Laikipia County. University of Nairobi

Nikolich-Žugich J (2018) The twilight of immunity: emerging concepts in aging of the immune system. Nat Immunol 19(1):10-19

Onyango CO, Njeru R, Kazungu S, Achilla R, Bulimo W, Welch SR, Cane PA, Gunson RN, Hammitt LL, Scott JAG (2012) Influenza surveillance among children with pneumonia admitted to a district hospital in coastal Kenya, 2007-2010. J Infect Dis 206(1):S61-S67

Pan W, Chen DS, Lu YJ, Sun FF, Xu HW, Zhang YW, Yan C, Fu LL, Zheng KY, Tang RX (2017) Bioinformatic prediction of the epitopes of Echinococcus granulosus antigen 5. Biomed Rep 6(2):181-187

Pereira G, Da Silva A, Do Nascimento S, De Mesquita J (2019) In silico analysis and molecular dynamics simulation of human superoxide dismutase 3 (SOD3) genetic variants. J Cell Biochem 120(3):3583-3598

Raghuvanshi D, Solanki V, Arora N, Hashmi F (2020) Comput Bioinform

Rakib A, Sami SA, Mimi NJ, Chowdhury MM, Eva TA, Nainu F, Paul A, Shahriar A, Tareq AM, Emon NU (2020) Immunoinformaticsguided design of an epitope-based vaccine against severe acute respiratory syndrome coronavirus 2 spike glycoprotein. Comput Biol Med 124:1967

Rashidian E, Gandabeh ZS, Forouharmehr A, Nazifi N, Shams N, Jaydari A (2020) Immunoinformatics approach to engineer a potent poly-epitope fusion protein vaccine against Coxiella burnetii. Int J Peptide Res Ther 26:1-11

Rosa DS, Ribeiro SP, Cunha-Neto E (2010) CD4+ T cell epitope discovery and rational vaccine design. Arch Immunol Ther Exp 58(2):121-130

Rosyda M, Chaerul D (2020) Physicochemical properties analysis of predicted SARS-Cov2 B-Cell epitope. Adv Med Sci Technol 1(1):27-34

Roy N (2020) Prediction of physicochemical properties of SARS$\mathrm{CoV}-2$ protein in Bangladesh

Sabbaghi A, Zargar M, Zolfaghari MR, Motamedi-Sedeh F, Ghaemi A (2021) Protective cellular and mucosal immune responses following nasal administration of a whole gamma-irradiated influenza A (subtype H1N1) vaccine adjuvanted with interleukin-28B in a mouse model. Adv Virol 166(2):545-557

Salaun C, Greaves J, Tomkinson NC, Chamberlain LH (2020) The linker domain of the SNARE protein SNAP25 acts as a flexible molecular spacer that ensures efficient S-acylation: role of the SNAP25 linker domain in S-acylation. J Biol Chem 295(21):7501-7515

Sanami S, Zandi M, Pourhossein B, Mobini G-R, Safaei M, Abed A Arvejeh PM, Chermahini FA, Alizadeh M (2020) Design of a multi-epitope vaccine against SARS-CoV-2 using immunoinformatics approach. Int J Biol Macromol 164:871-883

Sarkar B, Ullah MA, Araf Y (2020) A systematic and reverse vaccinology approach to design novel subunit vaccines against Dengue virus type-1 (DENV-1) and human Papillomavirus-16 (HPV- 16). Inform Med Unlocked 19:100343

Serwold T, Gonzalez F, Kim J, Jacob R, Shastri N (2002) ERAAP customizes peptides for MHC class I molecules in the endoplasmic reticulum. Nature 419(6906):480-483

Seyed N, Taheri T, Vauchy C, Dosset M, Godet Y, Eslamifar A, Sharifi I, Adotevi O, Borg C, Rohrlich PS (2014) Immunogenicity evaluation of a rationally designed polytope construct encoding HLA-A* 0201 restricted epitopes derived from Leishmania major related proteins in HLA-A2/DR1 transgenic mice: steps toward polytope vaccine. PLoS ONE 9(10):e108848

Shao W, Li X, Goraya MU, Wang S, Chen J-L (2017) Evolution of influenza a virus by mutation and re-assortment. Int J Mol Sci 18(8): 1650

Sharma, N., S. Patiyal, A. Dhall, A. Pande, C. Arora and G. P. Raghava (2020). "AlgPred 2.0: an improved method for predicting allergenic proteins and mapping of IgE epitopes." Briefings in Bioinformatics.

Sharma S, Kumari V, Kumbhar BV, Mukherjee A, Pandey R, Kondabagil K (2021) Immunoinformatics approach for a novel multiepitope subunit vaccine design against various subtypes of Influenza A virus. Immunobiology 226(2):152053

Singh G, Pritam M, Banerjee M, Singh AK, Singh SP (2020) Designing of precise vaccine construct against visceral leishmaniasis through predicted epitope ensemble: a contemporary approach. Comput Biol Chem 86:107259

Suresh P, Basu PK (2008) Improving pharmaceutical product development and manufacturing: impact on cost of drug development and cost of goods sold of pharmaceuticals. J Pharmaceut Innovat 3(3): $175-187$

Sussman JL, Lin D, Jiang J, Manning NO, Prilusky J, Ritter O, Abola EE (1998) Protein Data Bank (PDB): database of three-dimensional structural information of biological macromolecules. Acta Crystallogr D 54(6):1078-1084

Tahir ul Qamar M, Shokat Z, Muneer I, Ashfaq UA, Javed H, Anwar F, Bari A, Zahid B, Saari N (2020) Multiepitope-based subunit 
vaccine design and evaluation against respiratory syncytial virus using reverse vaccinology approach. Vaccines 8(2):288

Valkenburg SA, Josephs TM, Clemens EB, Grant EJ, Nguyen TH, Wang GC, Price DA, Miller A, Tong SY, Thomas PG (2016) Molecular basis for universal HLA-A* 0201-restricted CD8+ T-cell immunity against influenza viruses. Proc Natl Acad Sci USA 113(16):4440-4445

Van Zundert G, Rodrigues J, Trellet M, Schmitz C, Kastritis P, Karaca E, Melquiond A, van Dijk M, De Vries S, Bonvin A (2016) The HADDOCK2. 2 web server: user-friendly integrative modeling of biomolecular complexes. J Mol Biol 428(4):720-725

Vangone A, Rodrigues J, Xue L, van Zundert G, Geng C, Kurkcuoglu Z, Nellen M, Narasimhan S, Karaca E, van Dijk M (2017) "Sense and simplicity in HADDOCK scoring: lessons from CASP-CAPRI round 1." Proteins: structure. Funct Bioinform 85(3):417-423

Vita R, Mahajan S, Overton JA, Dhanda SK, Martini S, Cantrell JR, Wheeler DK, Sette A, Peters B (2019) The immune epitope database (IEDB): 2018 update. Nucleic Acids Res 47(D1):D339-D343

Wang W, Huang H, Bolon Y-T, Udell J, Vierra-Green C, Spellman SR, Maiers M, Kennedy C (2017) OR7 MiHAIP: Comprehensive pipeline to discover immunogenic minor histocompatibility antigens via whole genome sequences of HLA-matched donorrecipient pairs. Hum Immunol 78:8

Wiederstein M, Sippl MJ (2007) ProSA-web: interactive web service for the recognition of errors in three-dimensional structures of proteins. Nucleic Acids Res 35(suppl_2):W407-W410
Yamayoshi S, Uraki R, Ito M, Kiso M, Nakatsu S, Yasuhara A, Oishi K, Sasaki T, Ikuta K, Kawaoka Y (2017) A broadly reactive human anti-hemagglutinin stem monoclonal antibody that inhibits influenza A virus particle release. EBioMedicine 17:182-191

Yan Y, Tao H, He J, Huang S-Y (2020) The HDOCK server for integrated protein-protein docking. Nat Protocols 15(5):1829-1852

Yang J, Yan R, Roy A, Xu D, Poisson J, Zhang Y (2015) The I-TASSER Suite: protein structure and function prediction. Nat Methods 12(1):7-8

Yarmarkovich M, Warrington JM, Farrel A, Maris JM (2020) Identification of SARS-CoV-2 vaccine epitopes predicted to induce long-term population-scale immunity. Cell Rep Med 1(3):100036

Zhang S, Yin Y, Jones MB, Zhang Z, Kaiser BLD, Dinsmore BA, Fitzgerald C, Fields PI, Deng X (2015) Salmonella serotype determination utilizing high-throughput genome sequencing data. $\mathrm{J}$ Clin Microbiol 53(5):1685-1692

Zhou C, Zhou L, Chen Y-H (2012) Immunization with high epitope density of M2e derived from 2009 pandemic $\mathrm{H} 1 \mathrm{~N} 1$ elicits protective immunity in mice. Vaccine 30(23):3463-3469

Publisher's Note Springer Nature remains neutral with regard to jurisdictional claims in published maps and institutional affiliations. 